\title{
A combined community health worker and text messaging- based intervention for smoking cessation in India: Project MUKTI - A mixed methods study
}

Vittal Hejjaji', Aditya Khetan², Joel W. Hughes ${ }^{3}$, Prashant Gupta ${ }^{4}$, Philip G. Jones ${ }^{1}$, Asma Ahmed ${ }^{5}$, Sri Krishna Madan Mohan $^{6}$, Richard A. Josephson ${ }^{6}$

\begin{abstract}
INTRODUCTION We sought to evaluate the effectiveness of a community health worker (CHW) led smoking cessation intervention, supplemented by text messages, and tailored to an individual's readiness to quit.

METHODS We conducted a cluster randomized controlled trial (April 2018August 2019) in adult smokers residing in a semi-urban region of India. Participants in the intervention arm received CHW-led home visits and had the option of choosing to receive regular text messages. The dose and content of $\mathrm{CHW}$ counseling and text messages were tailored to the participant's readiness to quit. The control group received brief education only. Primary outcome was biochemically verified smoking cessation at the end of 12 months. Both intention-to-treat and as-treated analyses were performed.

RESULTS A total of 238 (mean age $43 \pm 12.3$ years, male $96.2 \%$ ) participants were enrolled; 151 (64\%) in the intervention arm and $83(35.4 \%)$ in the control arm. At 12 months, 31 (20.5\%) participants in the intervention arm and $9(10.8 \%)$ in the control arm quit smoking (absolute risk difference $=9.7 \%$; $R=1.69 ; 95 \%$ CI: $0.04-71.33, p=0.74)$. In the as-treated analysis, 17 (36.9\%) of the 46 participants who received optimal dose of the intervention quit smoking.

CONCLUSIONS CHW-led home-based counseling, supplemented by regular text messages, led to an increase in quit rates for smoking, especially among those exposed to a higher dose of the intervention. However, the difference in cessation rates was not statistically significant. Future studies should consider testing mobile application-based multimedia messaging with larger populations, as a supplement to $\mathrm{CHW}$-based counseling.
\end{abstract}

AFFILIATION

1 Department of Cardiovascular Medicine, Saint Luke's Mid America Heart Institute, University of Missouri Kansas City, Kansas, United States

2 Department of Cardiovascular Medicine, McMaster University Medical Center, Hamilton, Canada

3 Department of Psychology, Kent State University, Kent, United States

4 Department of Psychology, All India Institute of Medical Sciences, New Delhi, India

5 Department of Surgery, Ramaiah Medical College, Bangalore, India 6 Department of Cardiovascular Medicine Harrington Heart and Vascular Institute, University Hospitals Cleveland Medical Center - Case Western Reserve University, Cleveland, United States

\section{CORRESPONDENCE TO}

Vittal Hejjaji. Department of

Cardiovascular Medicine, Saint Luke's

Mid America Heart Institute, Saint Luke's

Hospital and University of Missouri Kansas City, 4401 Wornall Road, CV Research, 64111, Kansas City, Missouri, United States. Email:vhejjaji@saint-lukes.org

\section{KEYWORDS}

smoking cessation, community health worker, text messages, mobile health, lowand-middle-income country

Received: 30 November 2020

Revised: 26 December 2020

Accepted: 15 January 2021

\section{INTRODUCTION}

Smoking cessation is challenging but can be achieved through community and individual-level interventions ${ }^{1}$. While high income countries have successfully adopted both forms of interventions to reduce their burden of smoking, low- and middleincome countries (LMICs) have lagged in the adoption of individual-level interventions ${ }^{2,3}$. In India, although national public health policies have marginally improved cessation rates, individual-level 
smoking cessation counseling remains inadequate ${ }^{4,5}$. Smoking cessation support, delivered through readily available modes of communication and tailored to an individual's readiness to quit, can potentially increase smoking cessation rates in LMICs such as India.

Traditionally, community health workers ( $\mathrm{CHWs}$ ) in India have served as a bridge between patients and healthcare resources for the management of communicable, maternal, and newborn diseases ${ }^{6,7}$. In 2013, the government of India expanded the role of CHWs to promote healthy lifestyle habits such as smoking cessation, through community and homebased counseling sessions ${ }^{8}$. However, inadequate training, support, and the inherent difficulty in achieving behavior change have led to most CHWs feeling underprepared and overburdened, warranting additional external support ${ }^{9}$. Mobile health solutions in the form of text messages have been shown to be effective in promoting smoking cessation across a wide range of age groups and could potentially provide external support to enhance CHW efforts ${ }^{10,11}$. In 2015, the governmentinitiated Cessation program established a nationwide mobile health intervention that provides smoking cessation support via text messages to those willing to quit ${ }^{12}$. Although nearly 2 million individuals have registered for the program, most feel the need for additional in-person smoking cessation counseling as a supplement to the program's efforts ${ }^{13}$. A multicomponent intervention combining $\mathrm{CHW}$ and text messaging-based interventions could potentially have a synergistic effect in increasing smoking cessation rates.

Motivational interviewing (MI) is a patientcentered style of communication that has been shown to improve smoking cessation rates among those who have a low desire to quit and resist participation in cessation programs ${ }^{14,15}$. Additionally, MI has been effective in promoting smoking cessation across a wide range of age groups ${ }^{16}$. Although mostly used in high income countries by trained smoking cessation counsellors ${ }^{17}$, its use by lay health workers in LMICs has recently been shown to be promising ${ }^{18}$. Despite its effectiveness in improving smoking cessation rates, MI has previously not been evaluated for delivery by $\mathrm{CHWs}$ in India.

While the combined use of $\mathrm{CHW}$ and text messaging can potentially improve smoking cessation rates, there is no evidence to prove its effectiveness in LMICs. To fill this gap in evidence, we tested a Multi-Unit Kit for Tobacco cessation in India (MUKTI), incorporating CHW-led home visits and mobile text messages, tailored to an individual's readiness to quit, and hypothesized that such an intervention would result in higher cessation rates compared to brief education alone.

\section{METHODS}

\section{Study setting and population}

A detailed description of the study design has been previously given ${ }^{19}$. Briefly, between April 2018 and August 2019, we conducted a mixed methods two arm, parallel, pragmatic, cluster randomized controlled trial with qualitative analysis at the end of intervention in a semi-urban town, Dalkhola, West Bengal, India. The town has a population of about 20000 people with an estimated prevalence of smoking of $14 \%$ among adults $^{20,21}$. Using the voter list as a reference, the town was divided into geographical clusters consisting of equal population sizes. One female $\mathrm{CHW}$ was assigned to each geographical cluster who screened, enrolled, and later delivered the intervention.

We included all adult participants (aged 18-70 years) who self-reported their current daily use of cigarettes or bidis (hand rolled tobacco leaves) and had access to a mobile phone that had the capability to send and receive text messages. Bidis have a higher nicotine concentration $(21 \mathrm{mg} / \mathrm{g}$ vs $13 \mathrm{mg} / \mathrm{g}$ ) compared to conventional unfiltered cigarettes and are cheaper than cigarettes ${ }^{22}$. Participants who were bed-bound for any acute or chronic condition, had a significant disability such as being blind, deaf or intellectually disabled, preventing them from meaningfully participating in the trial were excluded from the study. Furthermore, the CHW had discretionary power to exclude any eligible participant based on her assessment of their ability to participate effectively in the trial and follow-up at the end of the intervention period.

\section{Study design and intervention}

The content and dose of the intervention were tailored to an individual's readiness to quit, determined using the trans-theoretical model of behavior change ${ }^{23}$. Based on the response to the question 'Are you ready 
to quit in the next 6 months?' at the beginning of the study, eligible participants were categorized into being pre-contemplative or contemplative to quitting smoking. The two main components of the intervention included CHW-led home-based smoking cessation counseling and culturally appropriate text messages delivered through mobile phones (Supplementary file Table 1). MI techniques were used by CHWs visiting participants who were precontemplative to the idea of smoking cessation.

CHWs in the study are individuals from the local community who are carefully selected through a written test and interview process and trained to provide smoking cessation counseling ${ }^{24}$. Once selected, each CHW underwent a one-week didactic session coupled with interactive role-playing to teach them MI techniques of communication. Additionally, each $\mathrm{CHW}$ had access to a detailed training booklet and a flipbook containing smoking cessation counseling material. The text messages were derived from a databank of messages that were previously used in a large smoking cessation clinical trial conducted in Australia ${ }^{25}$. Each of these messages were modified to make them culturally acceptable and translated into two local languages, Hindi and Bengali. The messages were then grouped into different sets, each targeting participants at different trans-theoretical stages of change (pre-contemplation, contemplation, determination, action, relapse, maintenance) and were automatically delivered to participants through a pre-defined algorithm. The algorithm was developed and customized using the open source software RapidPro ${ }^{26}$. Each delivery algorithm was activated by the user using a pre-defined code word, designated to their trans-theoretical stage of change. However, beyond activation of message sets using pre-defined code words, there was no further twoway communication or text message customization.

The intervention followed a dynamic process in which each participant had the ability to transition from one section of the intervention to the other based on their trans-theoretical stage of change, assessed by the CHW at every scheduled home visit. All participants received $\mathrm{CHW}$-led home visits at varying frequencies but the mobile health component was optional. Pre-contemplative participants received a CHW home visit every 2 months and 1 text message every week describing the benefits of smoking cessation. Contemplative participants received a CHW visit once a month for 3 months and were asked to set a quit date. The CHW visits and text messages focused on motivating and preparing the participant to quit smoking. After the quit date, the intervention was focused on avoiding relapse. Participants who relapsed received a CHW visit that focused on identifying potential reasons for relapse and reassessed their readiness to quit (Figures 1 and 2).

The MI sessions were unscripted and $\mathrm{CHWs}$ used various methods (open-ended questions, repeating back information, showing empathy and summarizing) of $\mathrm{MI}^{27}$. CHWs encouraged patient engagement in the conversation by developing a discrepancy between the patient's goals (such as health) and current behavior of smoking (building inner conflict). In addition, they explored ambivalence regarding smoking cessation, while avoiding argument or judgement. If participants expressed an interest in quitting, $\mathrm{CHW}$ visit and text message frequency were increased according to the contemplative workflow of the intervention.

Given that access to nicotine replacement therapy (NRT) was not widely available at the access site, it was made accessible through the $\mathrm{CHW}$ at market prices. At trial initiation, all control group participants received brief education through a single $\mathrm{CHW}$ led home visit regarding the benefits of smoking cessation. All outcomes were measured at the end of 12 months after the start of the intervention. The primary outcome of interest was 14-day selfreported abstinence, biochemically verified by measuring exhaled carbon monoxide ( $\mathrm{CO}$ ) levels. The use of exhaled CO is a commonly used metric that is easily obtained, but less accurate when compared to measurement of blood levels of nicotine metabolites ${ }^{28}$. A validated breath analyzer was used by the $\mathrm{CHW}$ to measure $\mathrm{CO}$ levels at the end of the intervention period and a cut-off point of $<10$ parts per million was used to confirm smoking cessation ${ }^{29}$. Secondary outcomes included variation in quit rates based on participant age and education status. At the end of the intervention, a sample of CHWs and participants were interviewed to understand their perspectives on the intervention. A semi-structured guide was used to facilitate an open discussion during 
Figure 1. Overall study design

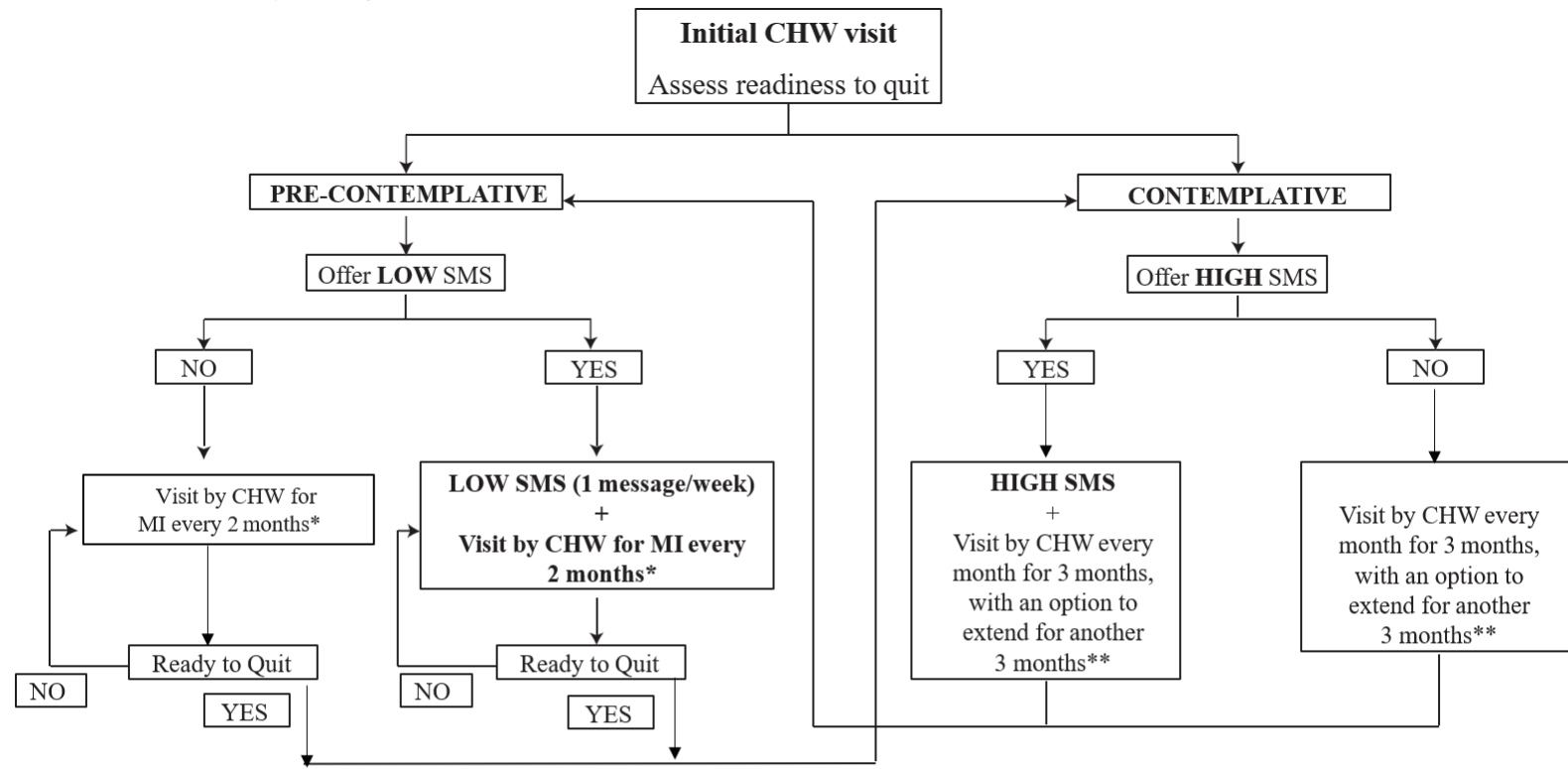

*Can visit every 4 months for participants who are

** For people who set a quit date,

heavily pre-contemplative, at $\mathrm{CHW}$ discretion

1) Phone call or personal visit $24-48$ hours before the quit date

2)Personal visit within 48 hours after the quit date

** For people who successfully quit

1) Monthly visits for 3 months, followed by visits every 2 months. After $>6$ months of being successfully quit, $\mathrm{CHW}$ can end visits at her discretion.

MI: motivational interviewing. CHW: community health worker. HIGH/LOW: high/low intensity messaging. SMS: short messaging service.

\section{Figure 2. Text-messaging format for contemplative participants (HIGH SMS)}

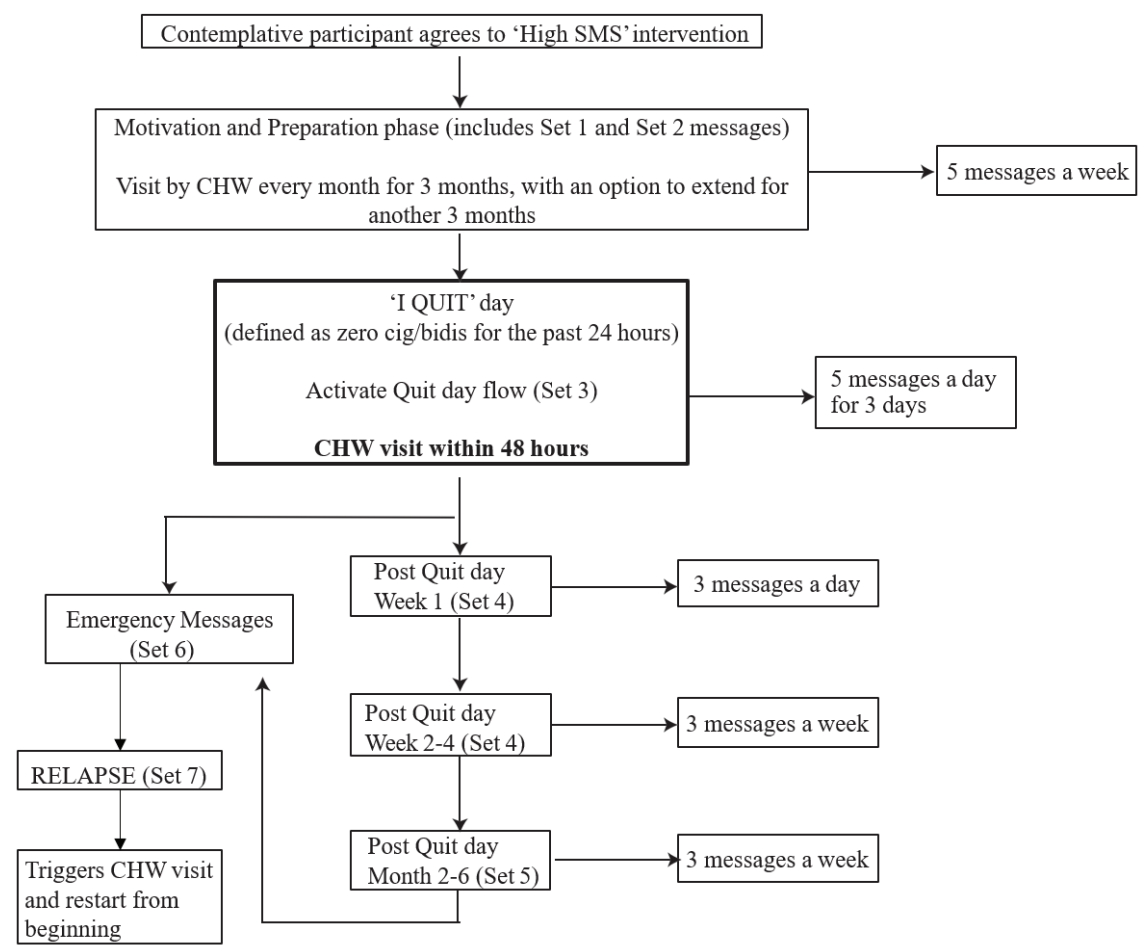

CHW: community health worker. SMS: short messaging service. 
the one-on-one phone interview conducted by one of the investigators (AK), a physician who is a native of the town, in the interviewee's local language. All discussions were audio-recorded and subsequently translated into the English language. Thematic analysis was conducted by two of the investigators ( $\mathrm{VH}$ and $\mathrm{AK}$ ) who evaluated all the $\mathrm{CHW}$ and participant interview transcripts independently, to identify common prevailing themes.

Prior to participation, all eligible participants were required to provide written informed consent. The institutional review board of University Hospitals Cleveland Medical Center in the USA and Society for the Promotion of Ethical Clinical Trials (SPECT), an independent review board in India, provided approval for this study. The trial was registered in the clinicaltrials.gov database (NCT03495622).

\section{Statistical analysis}

Sample size calculation for this study was based on the clustered pattern of delivering the intervention. It was calculated using a two-way significance level of 0.05 , a power of $80 \%$, minimum detection rate of $3.9 \%$ and an intra-class CI of 0.04 . An attrition rate of $30 \%$ was anticipated and the final estimated sample size was 560 participants, 35 per cluster across 16 clusters and two sites. Although the initial study protocol was designed to test the intervention at two sites, numerous site-level barriers to implementation led us to confine the study to a single site. Here, we report results from the single site, Dalkhola, which consisted of 8 clusters, 5 for intervention and 3 for control. Using an intention-to-treat analysis format, the Pearson's chi-squared test was used to compare the unadjusted and hierarchical logistic regression accounting for clustering at sites to compare adjusted cessation rates. Furthermore, we estimated all a priori secondary outcomes using similar regression tests and examined the cluster-level variability in uptake of the intervention and cessation rates. As sensitivity analysis, we compared cessation rates between groups in an as-treated format, among those who received an optimal dose of the intervention, defined as receiving $\geq 3 \mathrm{CHW}$-led home visits plus $\geq 5$ text messages. The analysis was performed using the R software v3.5.2.

\section{RESULTS}

Among 240 eligible participants, a total of 238 participants (mean age $43 \pm 12.3$ years; $96.2 \%$ male), 151 in the intervention group and 87 in the control group, provided informed consent. Half of the study cohort smoked bidis only and nearly $20 \%$ of the population smoked both cigarettes and bidis. At baseline, the majority of the participants had made no prior quit attempt and were predominantly $(84.6 \%)$ pre-contemplative to the idea of smoking cessation (Table 1). At the end of 12 months, a total of 4 participants in the control group died and 3 participants were lost to follow-up resulting in an overall retention rate of $97 \%$. The participants who died were excluded and those who were lost to follow-up were considered to be smokers at the end of the study period (Figure $3)^{28}$. With regard to the primary outcome, a total of $31(20.5 \%)$ participants in the intervention group and $9(10.8 \%)$ in the control group quit smoking with an absolute difference in cessation rate of $9.7 \%(R R=1.69$; 95\% CI: 0.04-71.33, $\mathrm{p}=0.74$ ) (Table 2).

Secondary outcomes examining quit rates based

Table 1. Baseline participant characteristics

\begin{tabular}{|c|c|c|c|}
\hline Characteristics & $\begin{array}{c}\text { All } \\
(\mathrm{N}=238) \\
n(\%)\end{array}$ & $\begin{array}{c}\text { MUKTI (Intervention) } \\
\left(\begin{array}{c}\mathbf{N}=151) \\
n(\%)\end{array}\right.\end{array}$ & $\begin{array}{l}\text { Brief advice (Control) } \\
\qquad \begin{array}{c}(\mathrm{N}=87) \\
\mathbf{n}(\%)\end{array}\end{array}$ \\
\hline \multicolumn{4}{|l|}{ Sociodemographic } \\
\hline Age (years), Mean (SD) & $43.0(12.3)$ & $41.8(12.0)$ & $43.0(12.2)$ \\
\hline Male & $225(96.2)$ & 147 (97.3) & $82(94.2)$ \\
\hline \multicolumn{4}{|l|}{ Religion } \\
\hline Hindu & $222(93.3)$ & $142(94.0)$ & 80 (91.9) \\
\hline Muslim & $12(5.0)$ & $5(3.3)$ & $7(8.0)$ \\
\hline Other & $4(1.7)$ & $4(2.6)$ & 0 \\
\hline Education level (school years), Mean (SD) & $9.31(3.77)$ & $9.48(3.7)$ & $9.30(3.7)$ \\
\hline
\end{tabular}


Table 1. Continued

\begin{tabular}{|c|c|c|c|}
\hline Characteristics & $\begin{array}{c}\text { All } \\
(\mathrm{N}=238) \\
n(\%)\end{array}$ & $\begin{array}{c}\text { MUKTI (Intervention) } \\
\left(\begin{array}{c}\mathbf{N}=151) \\
n(\%)\end{array}\right.\end{array}$ & $\begin{array}{l}\text { Brief advice (Control) } \\
\qquad \begin{array}{c}(\mathbf{N}=87) \\
n(\%)\end{array}\end{array}$ \\
\hline \multicolumn{4}{|l|}{ Work status } \\
\hline Gov. employee & $14(6.0)$ & $11(7.2)$ & $3(3.4)$ \\
\hline Private employee & $48(20.4)$ & $26(17.2)$ & $22(25.2)$ \\
\hline Self-employed & $95(40.4)$ & $91(60.2)$ & $5(5.7)$ \\
\hline Unemployed (able to work) & 39 (16.6) & $9(5.9)$ & $30(34.4)$ \\
\hline Unemployed (unable to work) & $13(5.5)$ & $3(1.9)$ & $10(11.4)$ \\
\hline Refused to answer & $16(6.8)$ & $4(2.6)$ & $12(13.7)$ \\
\hline Other & $10(4.2)$ & $5(3.3)$ & $5(5.7)$ \\
\hline \multicolumn{4}{|l|}{ Comorbidities } \\
\hline Prior history of respiratory disease & $7(3.0)$ & $6(3.9)$ & $1(1.1)$ \\
\hline Prior history of heart attack & $3(1.3)$ & $2(1.3)$ & $1(1.1)$ \\
\hline Prior history of stroke & $3(1.3)$ & $3(1.9)$ & 0 \\
\hline \multicolumn{4}{|l|}{ Smoking related information } \\
\hline Cigarettes/day*, Mean (SD) & $4.02(2.28)$ & $4.32(2.48)$ & $3.67(2.29)$ \\
\hline Bidis/day**, Mean (SD) & $8.60(4.63)$ & $9.00(4.93)$ & $7.91(4.63)$ \\
\hline Smoke cigarettes only & 65 (27.3) & $34(22.5)$ & $31(35.6)$ \\
\hline Smoke bidis only & $119(50.0)$ & $76(50.3)$ & $43(49.4)$ \\
\hline Smoke cigarettes and bidis & $43(18.0)$ & $30(19.8)$ & $13(14.9)$ \\
\hline Use pipe tobacco & $1(0.4)$ & $1(0.6)$ & 0 \\
\hline Prior quit attempt & $9(5.7)$ & $8(5.2)$ & $1(1.1)$ \\
\hline Contemplative stage of behavior change & $23(15.4)$ & $21(13.9)$ & $2(2.2)$ \\
\hline
\end{tabular}

*Estimated based on those who smoke cigarettes only. **Estimated based on those who smoke bidis only.

Figure 3. Consort diagram

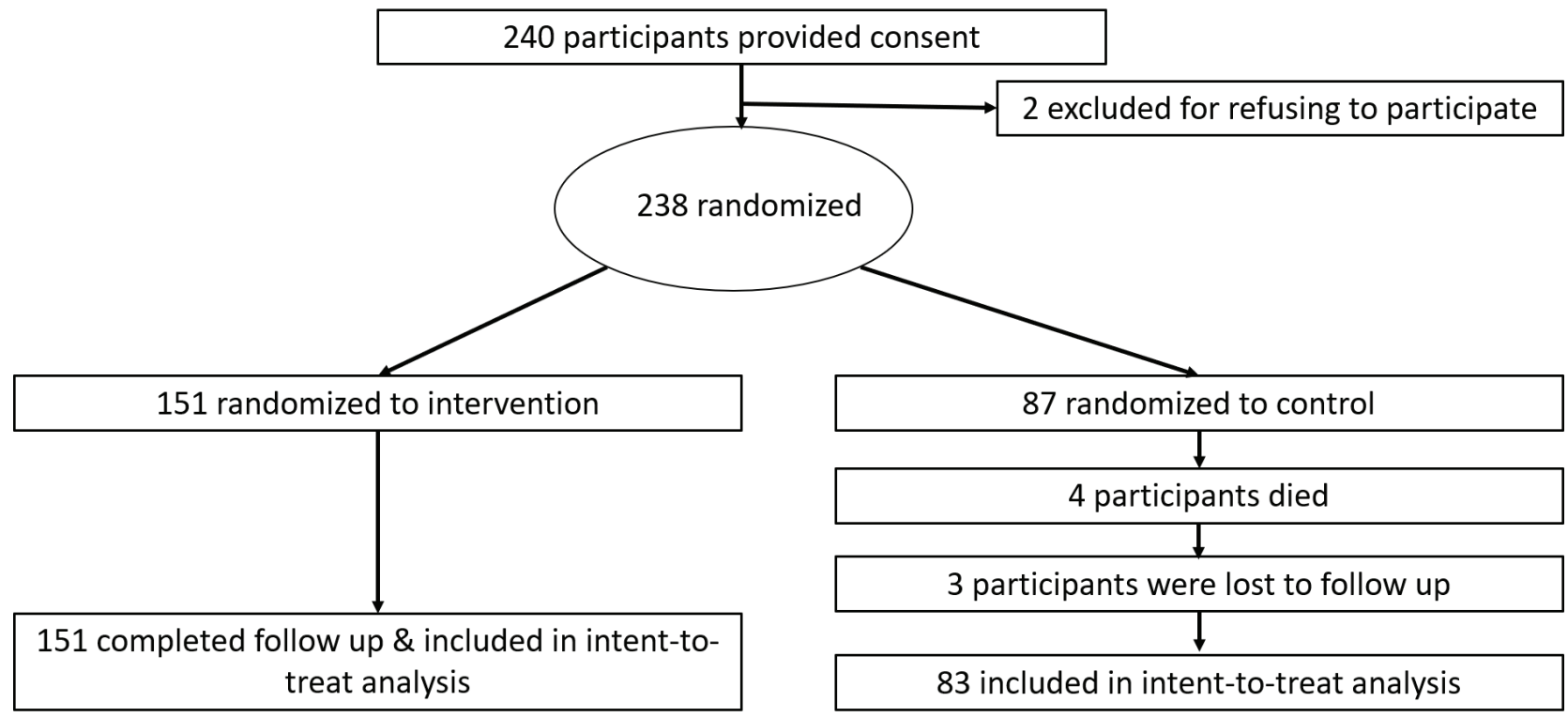


on participant age and education level are shown in Table 2. Participants aged $\leq 40$ years and those with $\geq 10$ years of schooling demonstrated greater smoking cessation rates compared to older and less educated individuals, however, the difference was not statistically significant. Furthermore, there was large cluster-level variation in the overall uptake of an optimal dose of the intervention (0-76.6\%) and

Figure 4. Cluster-level variability in intervention uptake and smoking cessation rates (data for 5 intervention clusters)

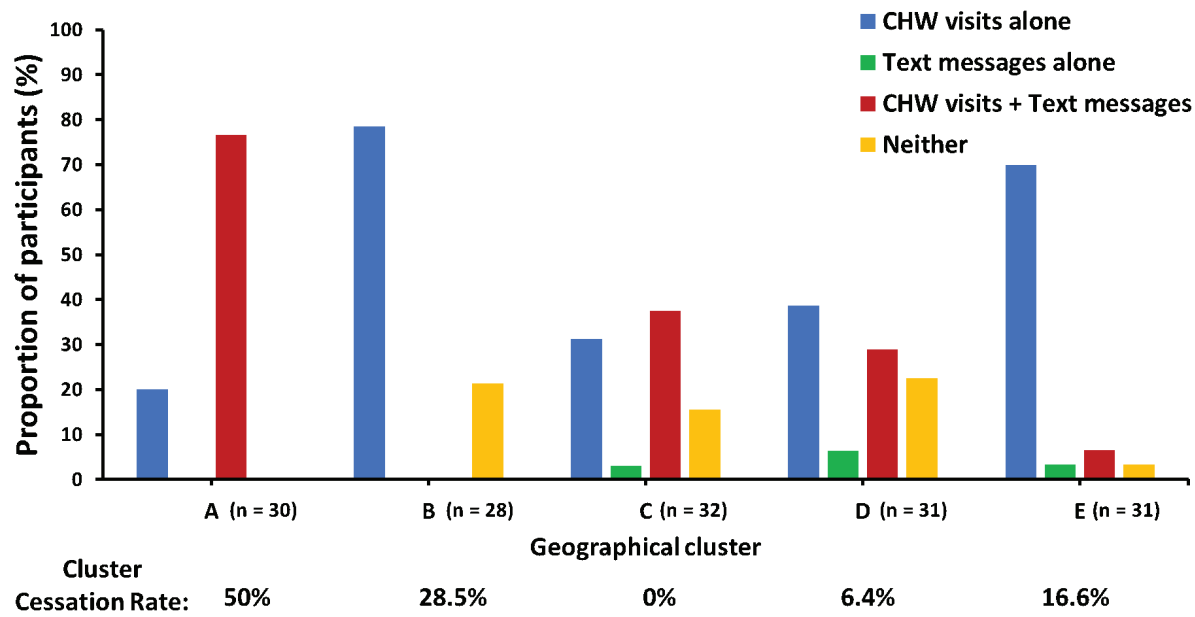

Community health worker visits alone: defined as those who received $\geq 3$ home visits plus $<5$. text messages. Text messaging alone: defined as those who received at least 5 messages plus $<3$ home visits. Community health worker visits plus text messages: defined as those who received at least 5 text messages plus $\geq 3$ home visits. Neither: is defined as those who received $<5$ text messages plus $<3$ home visits.

Table 2. Smoking cessation rates by intervention (MUKTI) and control

Characteristics

Intervention

arm

$n / \mathbf{N}(\%)$

Primary outcome (intent to treat analysis)

Biochemically verified 2-week point prevalence of smoking cessation at 12 months follow-up

$31 / 151(20.5) \quad 9 / 83(10.8)$

9.7

$1.69(0.04-71.3)$

0.74

Secondary outcome (intent to treat analysis)

Smoking cessation rates by age (years)

$<40$

$\geq 40$

$23 / 61(37.7)$
$5 / 75(6.7)$

2/21 (9.5)

$6 / 58(10.3)$

28.2

$5.41(0.11-268.4)$

0.33

Smoking cessation rates by years of schooling

$<10$

$7 / 49(14.3) \quad 3 / 31(9.7)$

4.6

$0.63(0.11-3.6)$

0.54

$\geq 10$

$20 / 83(24.1)$

$1 / 15(6.7)$

17.4

$2.88(0.06-17.8)$

0.99

As-treated analysis outcomes (dose of the intervention)
Optimal dose of the combined intervention

$\geq 3 \mathrm{CHW}$ visits plus $\geq 5$ text messages

Optimal dose of $\mathrm{CHW}$ visits alone

$\geq 3 \mathrm{CHW}$ visits plus $<5$ text messages

Optimal dose of text messaging alone

$<3 \mathrm{CHW}$ visits plus $\geq 5$ text messages

Suboptimal dose of the combined intervention

$<3 \mathrm{CHW}$ visits plus $<5$ text messages

$\begin{array}{rrrll}17 / 46(37.0) & 9 / 83(10.8) & 26.2 & 4.58(0.1-191.3) & 0.37 \\ 13 / 77(16.9) & 9 / 83(10.8) & 6.1 & 1.13(0.03-45.2) & 0.94 \\ 0 / 5(0.0) & 9 / 83(10.8) & \text { NA } & 0.00 & 0.99 \\ 1 / 23(4.4) & 9 / 83(10.8) & -6.4 & 0.50(0.01-43.0) & 0.72\end{array}$

RR: relative risk ratio. CHW: community health worker. NA: not applicable. 


\section{Table 3. Community health worker and participant perspectives}

\section{Community health worker interview highlights}

\section{Text messaging}

'Text messaging alone is unlikely to be effective but when used as a supplement to home-based counseling, increased my confidence to counsel patients.'

'Using WhatsApp messages instead of regular text messages will be more appealing.'

'Picture messages with more clear language would increase acceptability.'

\section{Community health worker-led counseling}

'Unlike diabetes and hypertension, smoking cessation requires more frequent home visits.'

'Age and family support are the most important factors that influence smoking cessation.'

'Customizing the counseling pattern to each individual's readiness to quit is most effective.'

CHW: community health worker.

cessation rates $(0-50 \%$; Figure 1$)$ with an intra-class CI of 0.05. There was negligible uptake of NRT, with only one participant obtaining NRT from the CHW.

In the as-treated analysis, uptake of the intervention was examined by estimating the proportion of participants who received an optimal dose of the intervention, defined as $\geq 3 \mathrm{CHW}$ visits and $\geq 5$ text messages. A total of 46 (30.4\%) participants received an optimal dose of the combined intervention. Among these participants, 17 (36.9\%) quit smoking with an absolute difference in cessation rate of $26.2 \%$ $(\mathrm{RR}=4.58$; 95\% CI: 0.11-191.29, $\mathrm{p}=0.37)$ (Table 2).

For the qualitative interviews, $3(60 \%) \mathrm{CHWs}$ - each achieving different cluster-level cessation rates $(0-50 \%)$, were chosen. All CHWs were female and had equal prior experience with home-based smoking cessation counseling. A total of $4(3 \%)$ male participants (mean age $27.2 \pm 2.5$ years; average 10 cigarettes/day) in the intervention arm volunteered to participate in an interview. Two of these participants had quit smoking by the end of the study, and only one had received an optimal dose of the intervention (both components at high doses). The important prevailing themes identified during the $\mathrm{CHW}$ and participant interviews are detailed in Table 3 .

\section{DISCUSSION}

In this mixed methods cluster randomized controlled

\section{Participant interview highlights}

'The messages are a good idea but WhatsApp is a better platform as pictures are more impactful.'

'I often ignored the messages as they were of little help.'

'I opted out of the text messaging option fearing the financial burden of receiving messages on a daily basis.'

'The CHW motivated me to quit smoking by telling me all the harmful effects of smoking:

'Ever since the CHW started to visit our home, my family members have been encouraging me to quit.'

'The CHW-led counseling sessions are more effective than text messages.'

trial, we evaluated the effectiveness of a multicomponent smoking cessation intervention involving CHW-led home-based counseling and text messages, tailored to an individual's readiness to quit. At the end of the study period, a greater number of participants in the intervention arm quit smoking compared to those in the control arm. However, the difference between the groups was not statistically significant, most likely due to low statistical power. Younger participants and those with 10 or more years of schooling had greater rates of smoking cessation. The potential impact of comorbidities on our outcomes is uncertain as only $13(5.5 \%)$ participants reported to have comorbidities. Marked cluster-level heterogeneity in the uptake of intervention components and smoking cessation rates were noticed. Additionally, within an as-treated analysis, a positive dose-response relationship was noticed among those who used both components of the intervention.

The relatively large proportion $(37 \%)$ of participants who quit smoking after receiving an optimal dose of the combined intervention suggests that a greater amount of support provided through CHW and text messages may be an effective solution to improve smoking cessation rates in India. However, the potential effectiveness of this finding is limited by the willingness of participants to receive the intervention at high doses. Only $30 \%$ 
of participants in the intervention group received an optimal dose of the intervention. The large clusterlevel variability in uptake of the intervention is most likely a reflection of the varying ability of $\mathrm{CHW}$ to engage participants. Furthermore, our results demonstrate that younger and more educated participants have higher smoking cessation rates - a finding consistent with other reports ${ }^{30,31}$. These observations can be explained by the fact that young, educated participants are high users of their mobile phones and may respond better to $\mathrm{CHW}$-led counseling efforts. The cessation rate of $10.8 \%$ in the control group is greater than the national average of $5 \%$ reported in 2015 - an unexpected finding ${ }^{4}$. The higher quit rates could reflect underlying secular trends due to the effect of national public health policies which have raised the cost of cigarettes and bidis, introduced graphic warnings on tobacco products and conducted mass media campaigns to promote smoking cessation ${ }^{32}$. Notably, qualitative interviews at the end of the trial demonstrated that CHW-led smoking cessation interventions are not only feasible but also a preferable mode of counseling. The home-based CHW model can improve access to individual-level counseling in low-and-middle-income countries such as India and facilitate additional support from family members.

Despite the government-led implementation of separate CHW and mobile-based smoking cessation interventions, there is limited evidence to support the effectiveness of either intervention in India, especially in the context of tailoring the intervention based on an individual's readiness to quit. A study evaluating the effectiveness of a single session of home-based $\mathrm{CHW}$-led cessation counseling, combined with training in vedic exercises, resulted in a $2 \%$ absolute increase in cessation rates in the intervention group over a follow-up period of 6 months ${ }^{33}$. Similarly, in a prospective evaluation of individuals enrolled in the text messages-based mCessation program in India, $77 \%$ of participants reported that the messages are extremely helpful to quit smoking, and $19 \%$ of participants quit 6 months after enrollment ${ }^{13}$. However, this analysis is limited by the lack of a control group and the selfreported assessment of smoking cessation status. To the best of our knowledge, ours is the first study to evaluate a combined CHW and text messaging-based intervention, substantially adding to the existing body of evidence. Although the difference between our two study arms was not statistically significant, a cessation rate of nearly $20 \%$ over one year in the intervention arm is similar to estimates from other national and international smoking cessation trials $^{10,11}$

Our study has several advantages. First, multiple theory-based smoking cessation interventions have primarily focused on tailoring the content of the intervention to an individual's readiness to quit (Web or mobile-based text messages $)^{34,35}$. By tailoring the dose and content of our intervention components, we were able to focus a higher dose of the intervention towards those who were willing to quit and simultaneously use evidence-based MI techniques to motivate those who were not. Such a strategy can potentially reduce $\mathrm{CHW}$ burden. Additionally, while most similar studies often exclude those who are not motivated to quit, we demonstrate that the $\mathrm{CHW}$ led model of motivational interviewing can be a potential solution to motivate individuals in quitting smoking. Second, the pragmatic study design made the text messaging component optional to the user and facilitated the dynamic transition of participants from one trans-theoretical stage of change to the other throughout the study period. The poor uptake of the mobile-based smoking cessation intervention in our study highlights the existing challenges of a mobile-based intervention in India, which has previously been shown to be ameliorated by promotion of text messaging based smoking cessation services by physicians ${ }^{36}$. Technology is continuously evolving and at the time of study inception, text messaging was the most commonly used mode of communication. However, the potential financial burden of receiving text messages and text messaging fatigue are some of the reasons cited by participants who opted out of the mobilebased component of the intervention. Given their widespread use and ability to send or receive picture and text messages at zero additional cost, multimedia messaging applications such as WhatsApp may be a preferred choice for future mobile health solutions in LMICs ${ }^{37}$. Third, this study highlights the feasibility of training lay $\mathrm{CHWs}$ in the use of MI techniques, a proven form of smoking cessation counseling in a clinic setting. A review of studies 
incorporating MI techniques to counsel smokers, demonstrated an overall weighted cessation rate of $17 \%$, similar to rates within our study cohort ${ }^{17}$. Fourth, this study demonstrates the acceptability of home-based CHW-led smoking cessation counseling in a semi-urban region of India. Due to the lack of adequate healthcare facilities in the region, such non-physician led interventions can be a sustainable and cost-effective solution to the large burden of smoking-related diseases. CHW-led counseling is particularly appealing as CHWs have the ability to spend adequate amount of time with the participant, provide intervention within the participant's home, and are able to involve family members who may be able to assist in achieving sustainable smoking cessation ${ }^{38}$. Finally, our qualitative interviews highlight the gaps and opportunities in combined smoking cessation interventions, which require further evaluation with multimedia mobile applications and larger sample sizes. Future interventions should focus on improving $\mathrm{CHW}$ training to enhance participant engagement, use multimedia applications instead of regular text messaging, and incorporate real-time data analysis to detect inadequate engagement and provide $\mathrm{CHW}$ supportive feedback.

\section{Limitations}

These results are to be interpreted in the context of several limitations. First, although our study protocol was designed to accommodate two sites within India, we were unable to implement our intervention at the second site in Jabalpur, India. This led to a lower than estimated sample size. Second, uptake of the intervention varied across clusters with many not receiving an optimal dose of the combined intervention. This was a consequence of the pragmatic study design, where text messaging was made optional to the participants. However, the positive dose-response relationship noticed in this trial highlights the potential benefits of combining these interventions and serves as a proof of concept for future clinical trials. Third, while all the CHW were female, the majority of our study population was male, which may have influenced our study findings. Future studies with male $\mathrm{CHW}$ s counseling male participants are needed to better understand the influence of CHWs' sex on outcomes. Fourth, the results from this single site intervention may have limited generalizability to other regions, especially in places where the use of mobile phones and access to a trained $\mathrm{CHW}$ are different. Fifth, we did not enroll consecutive households and did not collect information on number of participants screened (but subsequently excluded) for study participation. This may limit the generalizability of our findings. Finally, our study only focused on those who smoke cigarettes and/or bidis, and did not include users of smokeless tobacco products.

\section{CONCLUSIONS}

In this cluster randomized trial, we evaluated the effectiveness of a multi-component smoking cessation intervention, involving $\mathrm{CHW}$-led home-based counseling supplemented by mobile text messages. We found that, compared to brief advice alone, a greater number of participants in the intervention arm quit smoking at the end of 12 months. However, the difference was not statistically significant, likely due to inadequate sample size. Further studies are needed to evaluate the use of smoking cessation interventions that combine $\mathrm{CHW}$ counseling with multimedia-based messaging solutions such as WhatsApp.

\section{REFERENCES}

1. World Health Organization. WHO report on the global tobacco epidemic 2008. Geneva, Switzerland: World Health Organization; 2008. https://apps.who.int/iris/ bitstream/handle/10665/43818/9789241596282_eng. pdf?sequence=1. Accessed December 26, 2020.

2. Akanbi MO, Carroll AJ, Achenbach C, et al. The efficacy of smoking cessation interventions in low- and middleincome countries: a systematic review and meta-analysis. Addiction. 2019;114(4):620-635. doi:10.1111/add.14518

3. Kock L, Brown J, Hiscock R, Tattan-Birch H, Smith C, Shahab L. Individual-level behavioural smoking cessation interventions tailored for disadvantaged socioeconomic position: a systematic review and metaregression. Lancet Public Health. 2019;4(12):E628-E644. doi:10.1016/S2468-2667(19)30220-8

4. Mishra S, Joseph RA, Gupta PC, et al. Trends in bidi and cigarette smoking in India from 1998 to 2015 , by age, gender and education. BMJ Glob Health. 2016;1(1):e000005. doi:10.1136/bmjgh-2015-000005

5. Mohan P, Lando HA, Panneer S. Assessment of Tobacco Consumption and Control in India. Indian Journal of Clinical Medicine. 2018;9:1-8. doi:10.1177/1179916118759289

6. Patel AR, Nowalk MP. Expanding immunization coverage 
in rural India: a review of evidence for the role of community health workers. Vaccine. 2010;28(3):604-613. doi:10.1016/j.vaccine.2009.10.108

7. Wagner AL, Porth JM, Bettampadi D, Boulton ML. Have community health workers increased the delivery of maternal and child healthcare in India?. J Public Health. 2018;40(2):e164-e170. doi:10.1093/pubmed/fdx087

8. Ministry of Health and Family Welfare, Government of India. National Program for Prevention and Control of Cancer, Diabetes, CVD and Stroke (NPCDCS) . https:// www.nhp.gov.in/national-programme-for-preventionand-control-of-c_pg. Accessed December 26, 2020.

9. Abdel-All M, Abimbola S, Praveen D, Joshi R. What do Accredited Social Health Activists need to provide comprehensive care that incorporates non-communicable diseases? Findings from a qualitative study in Andhra Pradesh, India. Hum Resour Health. 2019;17(1):73. doi:10.1186/s12960-019-0418-9

10. Whittaker R, McRobbie H, Bullen C, Rodgers A, Gu Y. Mobile phone-based interventions for smoking cessation. Cochrane Database Syst Rev. 2016;4(4):CD006611. doi:10.1002/14651858.CD006611.pub4

11. Scott-Sheldon LA, Lantini R, Jennings EG, et al. Text Messaging-Based Interventions for Smoking Cessation: A Systematic Review and Meta-Analysis. JMIR Mhealth Uhealth. 2016;4(2):e49. doi:10.2196/mhealth.5436

12. Zahid. mCessation Programme - Quit Tobacco for Life. https://www.nhp.gov.in/quit-tobacco-aboutprogramme_mtl. Published December 23, 2015. Updated March 6, 2017. Accessed December 26, 2020.

13. Gopinathan P, Kaur J, Joshi S, et al. Self-reported quit rates and quit attempts among subscribers of a mobile text messaging-based tobacco cessation program in India. BMJ Innovations. 2018;4(4):147-154. doi:10.1136/bmjinnov-2018-000285

14. Catley D, Goggin K, Harris KJ, et al. A Randomized Trial of Motivational Interviewing: Cessation Induction Among Smokers With Low Desire to Quit. Am J Prev Med. 2016;50(5):573-583. doi:10.1016/j.amepre.2015.10.013

15. Gritz ER, Danysh HE, Fletcher FE, et al. Long-term outcomes of a cell phone-delivered intervention for smokers living with HIV/AIDS. Clin Infect Dis. 2013;57(4):608-615. doi:10.1093/cid/cit349

16. Heckman CJ, Egleston BL, Hofmann MT. Efficacy of motivational interviewing for smoking cessation: a systematic review and meta-analysis. Tob Control. 2010;19(5):410-416. doi:10.1136/tc.2009.033175

17. Lindson-Hawley N, Thompson TP, Begh R. Motivational interviewing for smoking cessation. Cochrane Database Syst Rev. 2015;(3):CD006936. doi:10.1002/14651858.CD006936.pub3

18. Louwagie GM, Okuyemi KS, Ayo-Yusuf OA. Efficacy of brief motivational interviewing on smoking cessation at tuberculosis clinics in Tshwane, South Africa: a randomized controlled trial. Addiction.
2014;109(11):1942-1952. doi:10.1111/add.12671

19. Khetan A, Hejjaji V, Hughes J, et al. Rationale and design of a study to test the effectiveness of a combined community health worker and text messaging-based intervention for smoking cessation in India (Project MUKTI). MHealth. 2019;5:15. doi:10.21037/mhealth.2019.05.04

20. Khetan A, Zullo M, Hejjaji V, et al. Prevalence and pattern of cardiovascular risk factors in a population in India. Heart Asia. 2017;9(2):e010931. doi:10.1136/heartasia-2017-010931

21. Asma S, Mackay J, Song SY, Zhao L, Morton J, Palipudi KM, et al. The GATS Atlas. Atlanta, GA: CDC Foundation; 2015. http://gatsatlas.org/. Accessed December 19, 2020.

22. Gupta PC, Asma S, eds. Bidi Smoking and Public Health. New Delhi, India: Ministry of Health and Family Welfare, Government of India; 2008. https://www.who.int/ tobacco/publications/prod_regulation/bidi_smoking_ public_health.pdf?ua=1. Accessed June 24, 2020.

23. Prochaska JO, DiClemente CG. The transtheoretical approach: Crossing traditional boundaries of therapy. Malabar, FL: Krieger Pub Co; 1994:193.

24. Khetan A, Patel T, Hejjaji V, et al. Role development of community health workers for cardiovascular disease prevention in India. Eval Program Plann. 2018;67:177183. doi:10.1016/j.evalprogplan.2018.01.006

25. Borland R, Balmford J, Benda P. Population-level effects of automated smoking cessation help programs: a randomized controlled trial. Addiction. 2013;108(3):618628. doi:10.1111/j.1360-0443.2012.04091.x

26. RapidPro [computer program]. New York, NY: UNICEF; 2020. https://community.rapidpro.io/. Accessed December 26, 2020.

27. Miller WR, Rollnick S. Motivational interviewing: Helping people change. 3rd ed. In: Applications of Motivational Interviewing Series. New York, NY: Guilford Press; 2012.

28. West R, Hajek P, Stead L, Stapleton J. Outcome criteria in smoking cessation trials: proposal for a common standard. Addiction. 2005;100(3):299-303. doi:10.1111/j.1360-0443.2004.00995.x

29. Vasthare R, Kumar S, Arron LYR. Carbon monoxide breath analyzers and its role in tobacco cessation: A narrative review of literature. J Int Oral Health. 2018;10(2):71-76. doi:10.4103/jioh.jioh_273_17

30. Sharma D, Goel S, Lal P. Education differential in relation to tobacco use and its predictors across different regions of India. Indian J Cancer. 2017;54(3):584-588. doi:10.4103/ijc.IJC_345_17

31. Parashar M, Singh M, Agarwalla R, Panda M, Pathak R. Predictors of intention to quit tobacco among construction site workers in Delhi, India. Indian J Psychiatry. 2017;59(2):208213. doi:10.4103/psychiatry.IndianJPsychiatry_368_16

32. Guindon GE, Fatima T, Li DX, et al. Visualizing data: Trends in smoking tobacco prices and taxes in India. Gates Open Res. 2019;3:8. doi:10.12688/gatesopenres.12894.1

33. Sarkar BK, West R, Arora M, Ahluwalia JS, Reddy KS, Shahab L. Effectiveness of a brief community outreach 
tobacco cessation intervention in India: a clusterrandomised controlled trial (the BABEX Trial). Thorax. 2017;72(2):167-173. doi:10.1136/thoraxjnl-2016-208732

34. Schlam TR, Baker TB. Interventions for tobacco smoking. Annu Rev Clin Psychol. 2013;9:675-702. doi:10.1146/annurev-clinpsy-050212-185602

35. Webb TL, Joseph J, Yardley L, Michie S. Using the internet to promote health behavior change: a systematic review and meta-analysis of the impact of theoretical basis, use of behavior change techniques, and mode of delivery on efficacy. J Med Internet Res. 2010;12(1):e4. doi:10.2196/jmir.1376

36. Basu S, Yadav P, Banerjee B, Yadav A. The effect of a clinic-based behavioral intervention in promoting enrolment in a text-message tobacco cessation program at a rural primary health clinic in Delhi, India. Tob Prev Cessation. 2020;6(May):1-7. doi:10.18332/tpc/120382

37. Bucher B. WhatsApp, WeChat and Facebook Messenger Apps - Global Messenger Usage, Penetration and Statistics. MessengerPeople. https://www.messengerpeople.com/ global-messenger-usage-statistics/. Published October 30, 2020. Accessed December 26, 2020.

38. Bittencourt L, Scarinci IC. Is there a role for community health workers in tobacco cessation programs? Perceptions of administrators and health care professionals. Nicotine Tob Res. 2014;16(5):626-631. doi:10.1093/ntr/ntt217

\section{ACKNOWLEDGMENTS}

The authors would like to thank Carole Decker and Christina Pacheco for their assistance in developing the qualitative questionnaire used to interview community health workers and participants. Additionally, we would like to thank Ron Borland for his guidance during the development of the text messages used in this trial.

\section{CONFLICTS OF INTEREST}

The authors have completed and submitted the ICMJE Form for Disclosure of Potential Conflicts of Interest and none was reported.

\section{FUNDING}

This work is supported by the Marwari Yuva Manch, a non-profit organization based in Dalkhola, West Bengal, India. VH is supported by the National Heart, Lung, and Blood Institute of the National Institutes of Health under award number T32HL110837.

\section{AUTHORS' CONTRIBUTIONS}

Conceptualization: VH, SKMM and RAJ. Methodology: VH, AK, JWH, PG, AA, SKMM and RAJ. Visualization: PG. Resources: $V H, J W H, P G$ and $A A$. Formal analysis: PGJ. Investigation: RAJ. Validation: $A K, P G$ and $A A$. Writing of original draft: $\mathrm{VH}$. Reviewing and editing of manuscript: All. Project administration: AK, PG and RAJ. Supervision: JWH, PGJ, SKMM and RAJ. Funding acquisition: $\mathrm{VH}$.

\section{PROVENANCE AND PEER REVIEW}

Not commissioned; externally peer reviewed. 